\title{
Intersections
}

Canadian Journal of Music

Revue canadienne de musique

\section{Catherine Rudent. 2011. L'album de chansons. Entre processus social et oeuvre musicale. Juliette Gréco, Mademoiselle K, Bruno Joubrel. Paris : Honoré-Champion, collection " Musique-Musicologie ». 274 p. ISBN 978-2-7453-2279-1 (couverture souple)}

\section{Danick Trottier}

Volume 32, numéro 1-2, 2012

URI : https://id.erudit.org/iderudit/1018590ar

DOI : https://doi.org/10.7202/1018590ar

Aller au sommaire du numéro

Éditeur(s)

Canadian University Music Society / Société de musique des universités canadiennes

ISSN

1911-0146 (imprimé)

1918-512X (numérique)

Découvrir la revue

Citer ce compte rendu

Trottier, D. (2012). Compte rendu de [Catherine Rudent. 2011. L'album de chansons. Entre processus social et oeuvre musicale. Juliette Gréco,

Mademoiselle K, Bruno Joubrel. Paris : Honoré-Champion, collection « Musique-Musicologie ». 274 p. ISBN 978-2-7453-2279-1 (couverture souple)]. Intersections, 32(1-2), 254-260. https://doi.org/10.7202/1018590ar

Copyright @ Canadian University Music Society / Société de musique des universités canadiennes, 2013
Ce document est protégé par la loi sur le droit d'auteur. L'utilisation des services d'Érudit (y compris la reproduction) est assujettie à sa politique d'utilisation que vous pouvez consulter en ligne.

https://apropos.erudit.org/fr/usagers/politique-dutilisation/ 
Catherine Rudent. 2011. L'album de chansons. Entre processus social et ouvre musicale. Juliette Gréco, Mademoiselle K, Bruno Joubrel. Paris: Honoré-Champion, collection «Musique-Musicologie». 274 p. ISBN 978-2-7453-2279-1 (couverture souple).

L'étude des musiques pop en musicologie francophone semble connaître un foisonnement extraordinaire ${ }^{1}$. Dans le cas de la France, le signe le plus patent en est la présence au sein de l'Observatoire musical français (OMF, basé en Sorbonne et dirigé par Danièle Pistone) du groupe de recherche Jazz, chanson, musiques populaires actuelles (JCMP), dont la création remonte à 2005. Les deux axes principaux du groupe sont l'analyse musicale et les problématiques sociologiques $^{2}$. Parmi les membres, on note des professeurs de Paris-Sorbonne à la réputation solidement établie: Laurent Cugny pour le jazz, Olivier Julien pour le rock anglophone et Hyacinthe Ravet pour la sociologie de la musique.

Vient compléter cette équipe Catherine Rudent, maître de conférence à l'Unité de formation et de recherche de musique et musicologie de Paris-Sorbonne depuis 2001. Les travaux de Rudent ont pour objet principal les musiques pop francophones actuelles, avec un intérêt marqué pour l'investigation sociologique et l'analyse musicale. Entre autres, les lecteurs d'Intersections ont pu la lire en 2006 dans un article consacré à la réception des voix de chanteuses et chanteurs québécois à la télévision française ${ }^{3}$. C’est à son premier livre sur l'étude des musiques populaires actuelles en France, avec Juliette Gréco, Mademoiselle K (i.e. Catherine Gierak) et Bruno Joubrel comme matières premières, que sont consacrées les prochaines lignes.

Dès le départ de l'ouvrage, la chercheure brosse un portrait sombre quant à la façon dont l'objet chanson est compris et étudié en France: les manquements pour comprendre comment une chanson fonctionne apparaissent criants. La difficulté de la situation est ainsi résumée: "Qui souligne l'importance, pour les compositeurs, les arrangeurs, et les interprètes d'abord, mais aussi pour les auditeurs plus extérieurs par la suite, des éléments musicaux dont se tisse le succès d'une chanson?" (p. 13). Partant de là, Rudent insiste sur l'écart qui reste à combler: «Or en France aujourd'hui, la formation musicologique qui permet de telles analyses [de l'objet lui-même à travers ses composantes structurelles, stylistiques et formelles] et l'intérêt pour les chansons françaises ont encore tendance à être largement disjoints: dès lors, peu de personnes sont susceptibles à la fois de vouloir et de pouvoir étudier les faits musicaux dont je parle, pourtant abondants et, pour qui veut bien prêter l'oreille, frappants et significatifs» (ibid.). Si l'on veut comprendre la chanson et les succès qu'elle remporte auprès du public, il faut l'étudier de l'intérieur, autant musicalement que socialement.

1 Les ouvrages se sont multipliés dans les dernières années comme en font foi des titres aussi différents que La Production de la culture. Le cas des musiques amplifiées en France de Jérôme Guibert (Irma Édition, 2006) ou Une histoire musicale du rock de Christophe Pirenne (Fayard, 2011).

2 http://www.omf.paris-sorbonne.fr/omf-articles.php3?id_rubrique $=98 \&$ id_article $=615$.

3 La page qui la présente sur le site de l'OMF donne une bonne idée de l'ampleur de ses recherches : http://www.omf.paris-sorbonne.fr/omf-articles.php3?id_rubrique $=116 \&$ \&id_article $=\% 20610$. 
C'est dans ce contexte que la chercheure propose un bref état des lieux de l'étude de la chanson, en soulignant autant la perspective interdisciplinaire (par exemple les travaux de Jérôme Guibert) que la perspective musicologique (notamment les travaux d'Olivier Julien). Aussi, et c'est tout à son honneur, Rudent ajoute à la liste, aux côtés des travaux de Serge Lacasse, ceux de Suzanne Lemieux et d'Audrée Descheneaux, montrant ainsi la perspective québécoise qui s'est dessinée ces dernières années; d'ailleurs, elle cite à de nombreuses reprises le livre de Gérald Côté, Processus de création et musique populaire. Un exemple de métissage à la québécoise (1998), l'approche processuelle de Côté n'étant pas étrangère à l'étude qu'elle propose.

Rudent cherche, dans ce livre, à démarquer la chanson en tant que genre, délimitation qui conduit à voir la pop comme point de départ, mais à distinguer la chanson comme phénomène musical et social. C'est une démarcation à laquelle elle tient, non pas tant pour isoler la chanson que pour en comprendre la spécificité et les logiques internes. À titre d'exemple, là où certains procédés musicaux jouent un rôle fondamental dans la conception d'une chanson rock, par exemple la puissance sonore, la vitesse et la façon dont les instruments comme la guitare électrique et la batterie sont travaillés, d'autres sont privilégiés en chanson: que l'on pense à la prégnance du texte, aux effets vocaux et à la manière de travailler l'arrangement.

D'ailleurs, le clivage rock/chanson se faufile tout au long du livre, entre autres en raison de l'album de Mademoiselle $\mathrm{K}$ à forte teneur rock, mais aussi parce que ce clivage, comme le résume Rudent (p. 198), est constitutif du champ phonographique français qui s'implante entre 1955 et 1963 et qui voit l'importation de phénomènes comme le rock'n'roll et l'émergence de nouvelles vedettes comme Johnny Hallyday. Selon Rudent, la dualité entre yé-yé et chanson à texte se prolonge dans les façons de faire actuelles. Ce constat est d'autant plus intéressant qu'il rejoint un clivage que l'on retrouve au Québec à la même époque (Yéyés versus chansonniers sera le titre de la revue musicale de 1965 créée par Robert Charlebois, Jean-Guy Moreau et Claudine «Mouffe» Monfette) et qui se répercutera jusqu'à nos jours, comme le montrent les études et les enquêtes menées sur la réception de la chanson québécoise, par exemple à travers la dichotomie «snobs/quétaines» (voir Ollivier 2006). Il est donc important de souligner au passage que plusieurs des faits rapportés par Rudent peuvent, à quelques différences près, s'appliquer à la chanson québécoise.

De quoi se compose l'ouvrage? L'introduction, sous-titrée "L'analyse d'albums entre logique musicale et logique sociale», ne peut être plus claire quant aux objectifs heuristiques que se fixe la musicologue: poser les conditions théoriques sous lesquelles peut s'étudier le phénomène populaire musical, regroupé ici autour de la notion de tradition phonographique. Ces conditions théoriques font intervenir deux traditions scientifiques: la sociologie et la musicologie. Ces deux traditions apparaissent clairement dans la façon dont Rudent a divisé son ouvrage : la première partie, qui s'intitule «Cadres», pose les balises théoriques de la recherche et les manières d'étudier la chanson; la seconde partie, «Processus», rend compte des différentes étapes mises en œuvre par les acteurs pour compléter un album de chansons; enfin la troisième 
partie, «Versions», analyse les chansons par l'intermédiaire des transformations qui conduisent au rendu sonore final. En ce sens, autant l'introduction que la première partie délimite l'objet d'étude en montrant que la chanson, approchée comme objet, doit aussi être rapportée à un contexte plus large: d'une part le travail en studio, d'autre part l'album.

C'est la raison pour laquelle Rudent revient sans cesse sur le processus collectif à la base du phénomène qu'elle étudie. La génétique des œuvres renvoie aux habitudes de travail et aux processus auxquels l'objet est soumis dans le temps. En ce sens, Rudent opte pour une approche de la chanson où le contexte montre la pluralité des relations entre les acteurs: l'objet chanson ne peut faire l'économie du processus qui a conduit les acteurs à le penser, à le concevoir et à déployer une foule d'opérations qui sont considérées pertinentes. Cette approche de l'objet artistique porte un nom en sociologie: il s'agit de l'approche processuelle, qui fait des œuvres d'art le résultat d'une longue chaîne d'interventions. La sociologie de l'interactionnisme symbolique d'Howard S. Becker (2010) incarne cette façon de concevoir l'objet artistique en misant sur les traces à la fois esthétiques et sociales que laisse l'œuvre d'art. Rudent s'en remet fréquemment à Becker pour appuyer cette perspective, tout en rapportant la chanson à un milieu de travail. Les acteurs auxquels elle s'intéresse sont nombreux, allant des compositeurs aux réalisateurs en passant par les interprètes, les arrangeurs, les ingénieurs, etc. Sans le mentionner ouvertement dans son livre, Rudent est fidèle à une perspective pragmatique fortement représentée en sociologie française ${ }^{4}$, entre autres par la place accordée aux acteurs, au contexte et à l'action, mais aussi aux formes de divergences et de négociations.

La perspective sociologique ne s'arrête pas là: elle est aussi incarnée dans une étude de terrain puisque les trois artistes qui fournissent à Rudent sa matière première lui ont ouvert (en partie ou en totalité selon les cas) leur laboratoire créatif, à savoir: Aimez-vous les uns les autres ou bien disparaissez (2003) de Juliette Gréco, Ça me vexe (2006) de Mademoiselle K et L'air du temps (2006) de Bruno Joubrel. Si Rudent fournit très peu de précisions sur le choix de ces trois artistes, on comprend que les conjonctures de l'étude doivent y être pour quelque chose, de même que la représentation idéale à laquelle elle parvient avec trois albums fort différents: autant d'un côté Juliette Gréco représente ce qui a force de stabilité en chanson, autant Mademoiselle K représente une nouvelle génération qui tente de renouveler les façons de faire (en tentant une cohabitation de procédés propres à la chanson et au rock), autant Bruno Joubrel, qui vit de son art de manière non professionnelle, apparaît comme l'artiste indépendant. Donc trois façons de faire, et trois manières bien différentes de s'entourer et de penser la conception d'un album.

4 Cette perspective pragmatique s'est imposée dans les années 1980 et 1990 à travers les travaux de plusieurs sociologues français. En atteste la répercussion qu'a eue De la justification. Les économies de la grandeur de Luc Boltanski et Laurent Thévenot (Gallimard, 1991) en sociologie de la musique et en sociologie de l'art. Pour ne donner qu'un exemple en sociologie de la musique, la perspective pragmatique se retrouve dans Figures de l'amateur. Formes, objets, pratiques de l'amour de la musique aujourd'hui, livre coécrit par Antoine Hennion, Sophie Maisonneuve et Émilie Gomart (La Documentation française, 200o). 
C'est à partir de ces trois artistes que l'investigation sociologique s'est définie: observations en contexte de studio d'une part, entretiens avec les acteurs d'autre part, mais à des différences près pour chacun des albums, l'investigation étant plus importante dans le cas de l'album de Mademoiselle $\mathrm{K}$ et plus en retrait dans le cas de l'album de Juliette Gréco (observation indirecte par l'intermédiaire de Laurent Cugny)—Gréco n’a pas été interviewée par Rudent contrairement à Katerine Gierak et Bruno Joubrel. D’ailleurs, Rudent cite abondamment les acteurs via l'information qu'elle a recueillie, ce qui est non seulement instructif mais fort appréciable pour appuyer les idées avancées.

Le déroulement de la deuxième partie du livre se comprend alors beaucoup mieux: Rudent s'intéresse aux processus qui conduisent à l'album final, véritable lieu de négociations et d'essais. D'ailleurs, c'est un des faits essentiels sur lequel Rudent insiste tout au long de l'ouvrage: les manières de faire sont constitutives des choix effectués par les acteurs et finissent par orienter la couleur générale de l'album, chaque acteur s'en remettant à un savoir-faire découlant d'une expérience particulière. Ce que cherche au fond à comprendre Rudent est la façon dont les acteurs travaillent: qui fait quoi et qui prend les décisions finales? Partant de ces questions, l'idée de règles communes intéresse la chercheure tout autant que les tensions en cours et les consensus obtenus. Car l'un des enjeux reste l'unité stylistique de l'album, sa fidélité à une certaine idée que l'on se fait de l'artiste, le nom associé à l'album apparaissant alors comme le dénominateur commun autour duquel les décisions se prendront.

Mais l'investigation de Rudent va plus loin puisque ce sont chacune des étapes du travail d'enregistrement qui sont disséquées, de l'enregistrement au mixage en passant par la réalisation. La façon dont la production est représentée ou le protocole d'enregistrement adopté prennent ici figurent d'enjeux quant à la portée esthétique que l'artiste veut donner à son album. Qu'est-ce qui fait en dernière instance que tout ce travail se tient? Comme le précise Rudent: «La question de l'interchangeabilité n'est donc finalement pas la plus pertinente ici. Il serait plus adéquat de parler d'un niveau de responsabilité que chacun s'accorde et se voit accorder par les autres, avec des nombreuses gradations d'importance qui échappent au filtre grossier des étiquetages professionnels usuels» (p. 143).

Quant à la troisième partie du livre, elle est la plus exhaustive et met en pratique la seconde tradition scientifique de Rudent évoquée plus haut, soit la musicologie avec l'analyse musicale en toile de fond. C'est ici que l'auteure comble une partie des retards constatés en début d'ouvrage en proposant une approche musicologique taillée sur mesure pour la chanson. Alors qu'elle avait opté pour une approche plus holistique de la chanson en s'attardant au processus dans les première et deuxième parties, cette fois-ci Rudent choisit une approche atomisée où la création de l'album est appréhendée en tant que parcours à travers lequel chacune des versions de chanson dévoile les choix compositionnels effectués. Par les maquettes et les versions, la musicologue en arrive à une meilleure compréhension des tournures stylistiques qui vont définir l'album en tant que tout. On entre alors dans les précisions du travail de studio, dans les responsabilités qui incombent à chacun et dans les façons 
de représenter les chansons. La construction globale de la chanson est au cœur de l'investissement musicologique tout autant que le travail d'arrangement et le style vocal des trois artistes.

C'est dans cette troisième et dernière partie que Rudent expose ses résultats musicologiques, en brossant entre autres un portrait stylistique des trois albums à la base de son étude tout en mettant de l'avant la perspective idiolectique (la contribution personnelle de chacun) qui se dégage des albums. Le style vocal demeure pour elle la composante essentielle pour saisir le travail des trois artistes et comprendre la façon dont la vocalité influence l'appréhension globale de la sonorité associée à l'artiste. La meilleure manière de comprendre ce style réside dans l'étude de la réalisation sonore en studio, moment où l'artiste avance d'essais en erreurs tout en développant des manières vocales qui lui sont propres.

En conclusion, souhaitons que cet ouvrage ait une incidence aussi importante que celui publié par Hennion en 1981, Les professionnels du disque. Une sociologie des variétés. Là où Antoine Hennion avait justement investigué la réalité de studio en tentant de comprendre les manières de faire et les choix des acteurs, et en ciblant comme fondamentales la «discomorphose» (qu'il approfondira plus tard, voir Hennion, Maisonneuve et Gomart, 2000), la relation artiste-public et la dimension commerciale, Rudent propose une perspective plus globale en reliant sans cesse l'objet chanson à l'album, puis en développant une perspective processuelle et un modèle théorique appropriés au cas de la chanson. Le souhait formulé ici quant à l'avenir de l'ouvrage repose sur plusieurs raisons. La première tient au fait que les ouvrages musicologiques portant sur pareil objet sont rares, de telle sorte que les bases posées dans cette étude influenceront les chercheurs à venir. La deuxième renvoie au positionnement théorique de Rudent, qui fait intervenir deux traditions scientifiques (musicologique et sociologique) tout en montrant la riche perspective qui s'en dégage. Enfin, il faut préciser que l'écriture est impeccable et que la terminologie employée est toujours justifiée, en plus d'un glossaire fort utile placé en fin d'ouvrage.

Cela ne veut pas dire que la recherche de Rudent soit sans lacunes. Une critique pouvant être formulée porte sur le statut des recherches existantes. $\mathrm{Si}$ la première partie du livre propose un bref état des lieux quant à l'étude musicologique de la pop et ses acteurs de premier plan, il aurait été profitable de voir les résultats de Rudent confrontés avec ceux de ses collègues. Par exemple, lorsqu'il est question du style vocal et du travail phonographique, plusieurs recherches de haut niveau auraient pu offrir des points de comparaison fort instructifs, ne serait-ce que pour le bilan de la recherche scientifique: les travaux de Serge Lacasse (2008) sur la phonographie vocale (et le modèle de transphonographie) viennent immédiatement à l'esprit, tout comme les propositions d'un Simon Frith (1996) sur l'expression de la voix pop ou d'un Philip Tagg (2000) sur le monocentrisme de la voix qui ressort de la projection sonore en studio.

Quant à la notion de valeur, elle est esquissée au fil des pages mais sans être véritablement approfondie. Elle est centrale puisque Rudent cherche à 
comprendre le succès commercial d'une chanson et les formes d'investissement dont elle fait l'objet. Cette notion fait intervenir un régime symbolique beaucoup plus large tout en nécessitant une mise en parallèle des formes de socialisation et des pratiques culturelles, ce qui est loin d'être le cas ici puisque l'approche est d'abord délimitée autour de la production de l'album. La tentation d'aller au-devant de la réception ressort à de nombreuses reprises dans l'ouvrage, comme si une telle réception ne pouvait être pensée qu'en termes d'horizon d'attente (pour reprendre l'expression de Jauss, 1978) eu égard à la tradition française de la chanson et à la trajectoire des artistes. Mais le succès commercial ne s'explique pas uniquement par la façon dont l'objet est conçu et par la notoriété des artistes. Les formes de grandeur dont la chanson est l'objet, l'attention médiatique qui la porte, la variation des conjonctures culturelles et les réseaux d'acteurs sont autant de réalités qui rapportent la chanson dans un espace de circulation beaucoup plus vaste. Si à la lecture de l'ouvrage l'on comprend mieux ce qui ressort comme important et pertinent dans la chanson, puis dans l'album, en revanche la notion de valeur démultiplie les contextes et les registres interpellés, comme le montrent les travaux de Frith (1996) : qu’on pense à la pluralité des formes de socialisation et des réseaux d'influence dans l'exercice du jugement esthétique pour arriver à qualifier et discriminer les musiques auxquelles chacun est exposé.

$\mathrm{Au}$ terme de la recherche, à quel bilan en arrive Rudent par rapport à la chanson actuelle française? Pour l'auteure, une véritable spécificité française dans la création d'albums se dégage de manière secondaire, entre autres en raison de l'utilisation de la langue française qui oblige les acteurs à faire des choix conséquents avec une tradition nationale. Autrement la chanson appartient à une réalité plus vaste qui est celle de la tradition phonographique, comme Rudent le rappelle au sujet des manières de faire que l'on retrouve autant en rock anglophone qu'en chanson française (elle donne l'exemple du «tâtonnement phonographique»). Elle en conclut alors: «Les manières d'opérer semblent dépendre de la génération, de la conception de la musique, ou encore des contraintes et des possibilités matérielles dans le cadre de telle ou telle création d'album, plus que d'une distribution géographique et spatiale des habitudes professionnelles» (p. 250). Voilà un constat qui défait bien des idées reçues et qui mérite à lui seul un détour dans le livre de Rudent.

\section{RÉFÉRENCES}

Becker, Howard S. 2010 [1988]. Les mondes de l'art. Paris: Flammarion.

Côté, Gérald. 1998. Processus de création et musique populaire. Un exemple de métissage à la québécoise. Paris: L’Harmattan.

Frith, Simon. 1996. Performing Rites. On the Value of Popular Music. Cambridge: Harvard University Press.

Hennion, Antoine. 1981. Les professionnels du disque. Une sociologie des variétés. Paris: Métailié.

Hennion, Antoine, Sophie Maisonneuve et Émilie Gomart. 2000. Figures de l'amateur. Formes, objets, pratiques de l'amour de la musique aujourd'hui. Paris: La Documentation française. 
Jauss, Hans Robert.1978. Pour une esthétique de la réception. Paris: Gallimard. Lacasse, Serge. 2008. "La musique pop incestueuse: une introduction à la transphonographie». Circuit 18, $\mathrm{n}^{\circ} 2: 11-26$.

Ollivier, Michèle. 2006. «Snob and quétaines: prestige and boundaries in popular music in Quebec». Popular Music 25, $\mathrm{n}^{\circ}$ 1: 97-115.

Rudent, Catherine. 2006. "La télévision française et les "voix québécoises" populaires: le trompe-l'œil d'un étiquetage médiatique». Intersections 27 , $\mathrm{n}^{\mathrm{o}} 1: 75-99$.

Tagg, Philip. 2000 [1991]. Fernando the Flute. Analysis of musical meaning in an Abba mega-hit, 2nd edition. Lima: Mass Media Music Scholars' Press.

DANick Trottier 